\title{
A comparative study of hard gelatin and hypromellose capsules containing a dry extract of senna (Cassia angustifolia) under controlled temperature and relative humidity
}

\author{
RENATA ARAUJO, M. SOARES, J. L. MAZZEI, MÔNICA RAMOS ${ }^{1}$ AND A. C. SIANI*
}

Department of Natural Products, Institute of Drug Technology, Oswaldo Cruz Foundation, 21041250 Rio de Janeiro, ${ }^{1}$ Department of Drugs and Medicines, Faculty of Pharmacy, Federal University of Rio de Janeiro, 21941902 Rio de Janeiro, Brazil

Araujo et al.: Gelatin and hypromellose capsules of senna extract

Dried natural extracts demand challenges to the production process, especially when encapsulated in hard shells to avoid compromising the integrity of the capsule and extract. The present study describes a preliminary comparative approach on the changes in properties of hard gelatin and hypromellose capsules containing senna (Cassia angustifolia Vahl) leaf dry extract under two storage conditions. Therefore, loss on drying, disintegration, resistance to breakage, water activity, and microbial contamination after 6 mo at $30 \% 65 \%$ RH and $40 \% / 75 \%$ RH were evaluated using Pharmacopoeia, AOAC and the manufacturer's methods. The contents of sennoside $A$ and sennoside $B$ in the capsules were assessed by HPLC. Fine-tosuperfine powder of the extract presenting $0.14 \%$ total ash and $2.9 \%$ loss on drying was applied. Uniformity of mass was repeatable independently of gelatin and hypromellose shells $(p>0.05$, $t$-test). The hypromellose capsules were more resistant to breakage than those of gelatin (maxima broken of 1/100 versus 42/100), in which the water content $(2.9 \%$ to $5.7 \%)$ and water activity $\left(0.33\right.$ to $\left.0.43 a_{w}\right)$ of the extract were intensively increased and, additionally, an acceptable increase in microbial contamination was observed. Compared to hypromellose shells, gelatin capsules did not maintain the same levels of both sennosides at $40 \% 75 \%$ RH, while sennoside B was lower. In conclusion, hypromellose performed better than gelatin to provide stable capsules, for resistance to breakage and disintegration unchanged, and offered higher protection to 
senna dry extract against humidity, microbial contamination and sennoside degradation. Overall, these preliminary results suggest the use of hypromellose encapsulation as a good alternative to gelatin in the production of medicinal capsules containing herbal dry extracts, mainly those with high hygroscopicity, and provide preliminary support for designing stability studies of capsules containing dry extracts that present high hygroscopicity.

Key words: Cassia angustifolia, senna extract, gelatin capsule, hypromellose capsule, hygroscopicity, hardness, sennoside A, sennoside B

The formulation of dried vegetable extracts has become increasingly important in a growing pharmaceutical market mainly after the inclusion of monographs of this type of raw material in pharmacopoeias ${ }^{[1]}$. Dry extracts of plant materials are obtained by drying fluid extracts with the aid of inert solid supports (adjuvants) to improve handling and manufacturing to achieve easier handling, easier transport and storage. Moreover, quality control also turns easier, as supported by a greater accuracy in the dosage of constituents and steadier procedures to assess the chemical, physical and microbiological conditions ${ }^{[2]}$. Capsules, generally those made of gelatin, have been the most common pharmaceutical formulation because their manufacture involves fewer steps, and they are more palatable to the consumer. Studies on capsules enclosing solid phytopharmaceuticals have rarely been reported; conversely, the properties and uses of gelatin capsules filled with (hydrophilic or hydrophobic) liquid have been periodically surveyed ${ }^{[3]}$. Although dried extracts are easy to handle, they still demand challenges to the production process, especially when hard gelatin capsules are used to formulate pharmaceuticals ${ }^{[4]}$. This situation is due to the hygroscopicity of most dry extracts, which causes them to absorb water when contacting the hard gelatin shell that is composed of protein polymers containing 13 to $16 \%$ moisture $^{[5]}$. This occurrence compromises the coating and thus the stability of the dry extract, in addition to reducing the process effectiveness. This effect can occur throughout the validity period of the medicinal product, which may cause changes in the dissolution profile and bioavailability ${ }^{[6]}$ of the herbal material, in addition to possibly increasing its susceptibility to microbial growth. Many studies on the use of a parentheses hydroxypropylmethylcellulose (hypromellose) as hard capsules have shown that they have greater flexibility and lower moisture retention levels (9\%) compared to other materials ${ }^{[7]}$. Moreover, better resistance to breakage by impact hypromellose capsules than those of gelatin has been reported elsewhere, mainly in dry powder capsules under lower moisture ${ }^{[8,9]}$. Therefore, the use of this cellulose-derived alternative has emerged as a promising technology to improve the production of herbal medicines by enhancing the encapsulation process efficiency ${ }^{[10]}$. The present preliminary study aims to comparatively evaluate the properties of hypromellose and gelatin capsules containing a senna (Cassia angustifolia Vahl) dry extract when submitted to two accelerated conditions over a period of $6 \mathrm{mo}$. The following parameters were evaluated: loss on drying, disintegration, susceptibility to breakage, water activity, microbial contamination, and content of sennoside A and sennoside B through high performance liquid chromatography (HPLC).

Senna leaf dry extract was acquired from Pharmanostra (Brazil). Starch was acquired from Cargill (Brazil). Capsugel (Pfizer Laboratory) kindly provided Transparent Coni-Snap gelatin (USA) and Vcaps Plus hypromellose (Mexico) capsules of size 0. Sennoside A (chromatographic grade) and sennoside B (analytical standard) were acquired from Carl Roth GmbH (Germany) and Sigma Chemical (USA), respectively. HPLC/UV-grade acetic acid and methanol were supplied by Tedia (USA) and ultrapurified water (resistivity $18.1 \mathrm{M} \Omega-\mathrm{cm}$ ) by Barnstead/Thermolyne (USA).

The senna dry extract applied in the present work was characterized physicochemically. The loss on drying was determined in independent quadruplicates by drying $1 \mathrm{~g}$ of extract in a VWR Scientific $1400 \mathrm{E}$ vacuum oven (USA) at $105^{\circ}$ for 2 hours until a constant weight was obtained in a Sartorius A200S (Germany) electronic balance of $0.1 \mathrm{mg}$ resolution. The total ash

This is an open access article distributed under the terms of the Creative Commons Attribution-NonCommercial-ShareAlike 3.0 License, which allows others to remix, tweak, and build upon the work non-commercially, as long as the author is credited and the new creations are licensed under the identical terms 
content (mg/g of air-dried extract) was determined in accordance with the Brazilian Pharmacopoeia general methods $^{[11]}$ using a Fornitec Mod 1646 electrical furnace (Brazil). The particle size analysis was performed in triplicate by the traditional homogeneity test for pharmaceutical powders ${ }^{[11]}$, from mesh sizes of 180, 150, 106, 75, and $63 \mu \mathrm{m}$ under a Retsch AS 200 (Germany) vibratory shaker.

The hard capsule shells of both gelatin and hypromellose were independently filled with the senna dry extract (550 mg) using starch as the excipient in a manual encapsulator (Capsutec, Brazil). The average weight of capsules was determined from twenty units based on the method described by Brazilian Pharmacopoeia ${ }^{[11]}$ and the United States Pharmacopoeia (USP) ${ }^{[12]}$. Loss on drying was determined at $105 \pm 2^{\circ}$ for 3 hours in a Lindberg/Blue M MO1490A-1 temperature-controlled oven (USA). According to the testing guidelines for new drug products ${ }^{[13]}$, three $60-\mathrm{mL}$ bottles of highdensity polyethylene (Vepakum, Brazil), containing 60 filled capsules each, were stored for $6 \mathrm{mo}$ in climatic Darwin Chamber PH052 (USA) at controlled temperature and relative humidity $(\mathrm{RH})$ of $30 \% 65 \%$ $\mathrm{RH}$ and of $40^{\circ} / 75 \% \mathrm{RH}$. According to the USP method $^{[12]}$, disintegration was assessed by using one capsule (empty or filled) of gelatin or hypromellose in $900 \mathrm{ml}$ of distilled water operated at $37^{\circ}$ in a Sotax DT2 apparatus (Switzerland) at 20-32 cycles/min. Six determinations were performed. Mechanical strength was evaluated using the Capsugel tube test method ${ }^{[10]}$ on empty and pre-emptied groups of 100 capsules to evaluate the resistance to breakage. A tightly closed (empty) individual capsule was placed under a firm, flat surface, and a trigger activated the drop of a 100 $\mathrm{g}$ stainless steel block onto the surface from a height of $8 \mathrm{~cm}$. At the end, any holes, cracks, or small breaks were identified for each capsule. The water activity of empty and filled capsules was determined at $25^{\circ}$ in an Aqualab Water Activity Meter Mod 4TE (USA) using the chilled-mirror dew point technique recommended by AOAC International ${ }^{[14]}$. Microbiological assays were conducted according to the USP method ${ }^{[12]}$. Samples of empty or filled with senna leaf dry extract $(10 \mathrm{~g})$ capsules were homogenized aseptically in $90 \mathrm{~mL}$ of Tryptic Soy Broth medium + casein broth, peptone, lecithin and Tween 80 . The material was then placed in a water bath at $35^{\circ}$ under mild stirring to complete the capsule dissolution. Aliquots of this solution were transferred to sterile Petri plates and spread together with the respective medium for the growth of aerobic bacteria, molds and yeasts. After solidifying at room temperature, they were incubated under conditions that were specific for each microorganism. The number of colony-forming units (CFU)/g of each sample was determined after the incubation period by counting the number of colonies. Pathogenic investigation was conducted for Staphylococcus aureus, Pseudomonas aeruginosa, Escherichia coli and Salmonella spp. The content of sennoside A and sennoside $B$ in the capsules was determined by an HPLC method according to the literature ${ }^{[15]}$, with a few modifications. The LC-10AVP system (Shimadzu Co., Kyoto, Japan) consisted of two LC-10AD pumps, a DGU-12A degasser, an SIL-10AD autosampler, a CTO-10A column oven and an SPD-M10A photodiode array detector, with scanning at 200-800 $\mathrm{nm}$ at $1 \mathrm{~nm}$ resolution. The data analysis was performed using CLASS-VP v.6.13 SP2 software (Shimadzu). The runs were performed by injecting $20 \mu \mathrm{L}$ at $25^{\circ}$ in a $150 \times$ $4.6 \mathrm{~mm}$ Supelcosil LC-18 $5 \mu \mathrm{m}$ column (Supelco, Bellefonte, USA) connected to a Supelguard LC18 guard column ( $2 \mathrm{~cm}$, Supelco). The mobile phase consisted of methanol/water/acetic acid at 20:80:0.1\% $\mathrm{v} / \mathrm{v} / \mathrm{v}$ (pump A) and at 80:20:0.1\% v/v/v (pump B) programmed on gradient elution: $0-5 \mathrm{~min}, 20 \% \mathrm{~B}$; 5-25 min, 20-100\% B; 25-32 min, 100\% B; 32-35 min, $100-20 \% \mathrm{~B}$; and $35-50 \mathrm{~min}, 20 \% \mathrm{~B}$. The flow rates were as follows: $0-25 \mathrm{~min}, 0.6 \mathrm{~mL} / \mathrm{min} ; 25-32 \mathrm{~min}$, $1.0 \mathrm{~mL} / \mathrm{min}$; $32-35 \mathrm{~min}, 0.6 \mathrm{~mL} / \mathrm{min}$ holding for $15 \mathrm{~min}$. The chromatograms were monitored at $275 \mathrm{~nm}$. Peaks at 18.5 and 21.1 min were identified as sennoside $B$ and sennoside A, respectively, based on the retention times of the standards and similarity of their UV spectra. All sample and standard solutions were injected in triplicate. The capsule contents were homogenized, and $250 \mathrm{mg}$ aliquots were separately weighed and placed into $5.0 \mathrm{~mL}$ volumetric flasks, followed by the addition of $3 \mathrm{~mL}$ of $7: 3$ methanol/water, sonication for $5 \mathrm{~min}$, dilution to the required volume with 7:3 methanol/water, and filtration through centrifugal filter devices (Durapore, PVDF membrane, $0.2 \mu \mathrm{m}$ ) prior to the injections. Sennoside A and sennoside B were quantified based on their respective peak areas and calibration curves, which were built using the results of triplicate injections, under equal conditions, of standard anthraquinone acid solutions at 2.0, 5.0, 10, 20, and 50 $\mu \mathrm{g} / \mathrm{mL}$ in $7: 3 \mathrm{methanol} /$ water.

The senna extract presented values for loss on drying $(2.9 \%)$ in compliance with the pharmacopoeia limit of $5 \%{ }^{[1]}$, considering senna leaf dry extract. Total ash content $(0.14 \%)$ is not still regulated in pharmacopoeias 
for senna leaf dry extracts but was much lower than $12 \%$, which is the limit for senna leaves ${ }^{[11]}$. Only $2.63 \%$ of the total mass of the extract was retained in the 180$\mu \mathrm{m}$ mesh sieve, which classifies it as a fine powder ${ }^{[1]}$.

The average weights for gelatin- and hypromellosefilled capsules, were $0.671 \pm 0.0043$ and $0.672 \pm 0.0090$ $\mathrm{g}$, respectively, without a significant difference $(\mathrm{p}>>$ 0.05 , t-test) and with theoretical contents corresponding to 556 and $554 \mathrm{mg}$ of extract per capsule, respectively. In fact, the very low relative standard deviations (RSDs $\leq 1.34 \%$ ) illustrates the excellent repeatability of the encapsulation procedure and, consequently, of the content uniformity. This result is well below the limit of variation recommended by the Brazilian Pharmacopoeia ${ }^{[11]}$, which is $\pm 7.5 \%$ of the theoretical weight for capsules with a capacity above $300 \mathrm{mg}$. However, statistical variations in the weights were broader for hypromellose capsules than those of gelatin ( $\mathrm{F}$ test $=4.38, \mathrm{p}<0.01)$. The extract-filled gelatin capsules poorly exhibited resistance to breakage, particularly when subjected to $40^{\circ} / 75 \% \mathrm{RH}$ when more than $40 \%$ showed some cracking (Table 1). The slow and continuous gelatin dehydration in the presence of the hygroscopic extract might have contributed to this result. Moreover, crosslinking and oxidative deamination of gelatin molecular structures induced by high temperatures are well known and can contribute to lower resistance to breakage ${ }^{[16]}$. The results of gelatin capsules of both conditions exceeded the manufacturer's acceptable limit for brittleness, which is six broken

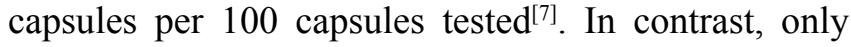
one out of every 100 capsules of hypromellose was susceptible to breakage. Based on composition, hypromellose capsules seem to be softer than those of gelatin under ambient conditions ${ }^{[17]}$, however, the wellknown higher susceptibility to breakage over lower moisture by gelatin than hypromellose in capsules for inhalers after releasing the filling ${ }^{[8,9]}$ can explain the enhanced effect by the hygroscopic fill formulation on this difference, as observed herein.

The high loss of hardness clearly affected the extract encapsulated in gelatin shells under $40^{\circ} / 75 \% \mathrm{RH}$ (Table 1$)$, in which maximal humidity $(5.7 \%)$ and water activity $\left(0.43 a_{w}\right)$ were precisely observed. The variable water activity between the dry extract and the content of the capsules can consecutively contribute to the microbial growth rate and enzymatic activity ${ }^{[12]}$. In fact, even though the total aerobic bacteria, molds and yeasts were found below the specification limits (1000, 100 and $100 \mathrm{CFU} / \mathrm{g}$, respectively) ${ }^{[12]}$. Some CFUs were observed only for filled gelatin capsules after $6 \mathrm{mo}$ : $16 \mathrm{CFU}$ of aerobic bacteria per gram at $40^{\circ} / 75 \%$ $\mathrm{RH}$ and 12 and $34 \mathrm{CFU}$ of molds and yeasts per $\mathrm{g}$ at $30 \% 65 \% \mathrm{RH}$ and $40 \% 75 \% \mathrm{RH}$, respectively. S. aureus, P. aeruginosa, E. coli and Salmonella spp. were absent. The contents of sennoside A and sennoside B under the programmed conditions were assessed through quantitative HPLC ${ }^{[15]}$. The excellent linearity of the calibration curves can be visually checked (fig. 1, r $>0.999)$, and good repeatability $(<5 \%$ RSD) for the

TABLE 1: PROPERTIES OF SENNA LEAF DRY EXTRACT AND CAPSULES BEFORE AND FILLING BY THE SENNA LEAF DRY EXTRACT AFTER 6 MONTHS AT TWO CONDITIONS

\begin{tabular}{|c|c|c|c|c|c|c|}
\hline Capsule & Condition & $\begin{array}{c}\text { Capsule shell or its } \\
\text { content }\end{array}$ & Loss on drying (\%) & $\begin{array}{l}\text { Disintegration } \\
\text { (min) }\end{array}$ & $\begin{array}{c}\text { Susceptibility to } \\
\text { breakage (units/100) }\end{array}$ & $a_{w}\left(25^{\circ}\right)$ \\
\hline \multirow[t]{9}{*}{$\bar{G}$} & Initial & Empty & 14.4 & 2.51 & 0 & 0.41 \\
\hline & $30^{\circ} / 65 \% \mathrm{RH}$ & Empty & 14.5 & 2.53 & 0 & - \\
\hline & & Filled capsule & . & 4.35 & - & - \\
\hline & & Shell after emptying & 13.2 & - & 7 & 0.37 \\
\hline & & Content after emptying & $3.5^{\mathrm{a}}$ & - & - & $0.35^{\mathrm{b}}$ \\
\hline & $40^{\circ} / 75 \% \mathrm{RH}$ & Empty & 15.1 & 4.53 & 4 & - \\
\hline & & Filled capsule & & 4.35 & - & - \\
\hline & & Shell after emptying & 12.3 & - & 42 & 0.34 \\
\hline & & Content after emptying & $5.7^{\mathrm{a}}$ & - & - & $0.43^{\mathrm{b}}$ \\
\hline \multirow[t]{9}{*}{$\mathrm{H}$} & Initial & Empty & 4.4 & 5.11 & 0 & 0.36 \\
\hline & $30^{\circ} / 65 \% \mathrm{RH}$ & Empty & 4.4 & 5.23 & 0 & - \\
\hline & & Filled capsule & - & 5.47 & - & - \\
\hline & & Shell after emptying & 4.6 & - & 0 & 0.36 \\
\hline & & Content after emptying & $3.1^{\mathrm{a}}$ & - & - & $0.34^{\mathrm{b}}$ \\
\hline & $40^{\circ} / 75 \% \mathrm{RH}$ & Empty & 4.3 & 5.25 & 0 & - \\
\hline & & Filled capsule & - & 6.02 & - & - \\
\hline & & Shell after emptying & 4.5 & - & 1 & 0.35 \\
\hline & & Content after emptying & $3.6^{\mathrm{a}}$ & - & - & $0.37^{\mathrm{b}}$ \\
\hline
\end{tabular}

Capsules: $\mathrm{G}$ is gelatin and $\mathrm{H}$ is hypromellose. Initial values for the senna leaf dry extract: ${ }^{\mathrm{a}} 2.9 \%$ and ${ }^{\mathrm{b}} 0.33$ 


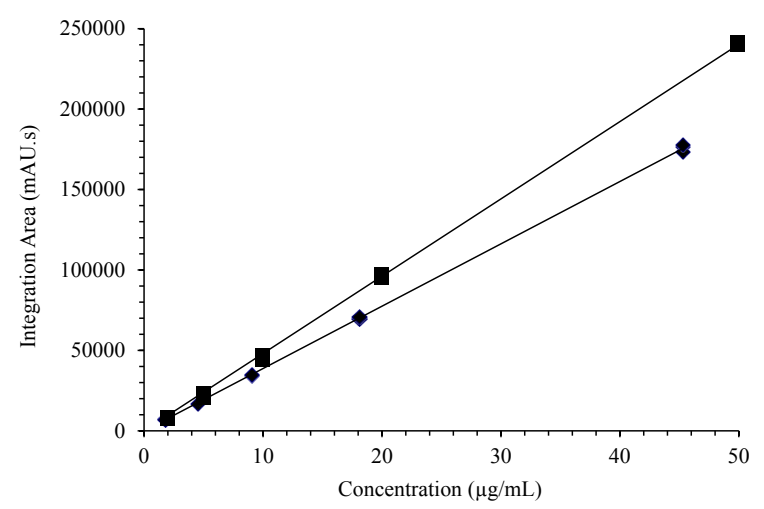

Fig. 1: Calibration curves of standard sennoside A (diamond, lower) and sennoside B (square, upper) in analysis by HPLC

TABLE 2: QUANTITATION OF SENNOSIDES IN THE CONTENT OF CAPSULES FORMULATED WITH SENNA LEAF DRY EXTRACT

\begin{tabular}{lccc}
\hline Capsule & \multirow{2}{*}{ condition } & \multicolumn{2}{c}{$\mathrm{mg} / 100 \mathrm{~g}$ of capsule content } \\
\cline { 3 - 4 } Gell & & Sennoside $\mathrm{A}$ & Sennoside $B$ \\
Gelatin & $30^{\circ} / 65 \% \mathrm{RH}$ & $93 \pm 6^{\mathrm{a}}$ & $21 \pm 1^{\mathrm{a}}$ \\
& $40^{\circ} / 75 \% \mathrm{RH}$ & $77 \pm 1^{\mathrm{b}}$ & $2 \pm 1^{\mathrm{b}}$ \\
Hypromel- & $30^{\circ} / 65 \% \mathrm{RH}$ & $95 \pm 4^{\mathrm{a}}$ & $22 \pm 1^{\mathrm{a}}$ \\
lose & $40^{\circ} / 75 \% \mathrm{RH}$ & $97 \pm 5^{\mathrm{a}}$ & $17 \pm 1^{\mathrm{c}}$ \\
\hline
\end{tabular}

RH: Relative humidity. "Mean and standard deviation, determined in triplicate by HPLC after 6 mo at each condition and capsule shell. Values obtained from the same sennoside with different superscript letters present significant differences $(p<0.01$, ANOVA and Tukey's test)

integration area was achieved at all concentration levels in compliance with guideline recommendations for validation of analytical procedures ${ }^{[18]}$. The regression equations were $y=3,891,600 x-577$ and $y=4,260,240 x$ +521 for sennoside A and sennoside B, respectively, in which $x$ is the concentration of anthraquinone in $\mu \mathrm{g} / \mathrm{ml}$ and $y$ is the mean integrated area. The significance of the regression was checked by ANOVA, confirming that the slope and intercept values were different from zero (F-calculated $>$ F-tabulated, $\mathrm{p}<0.05)$. As expected, more drastic conditions $\left(40^{\circ} / 75 \% \mathrm{RH}\right)$ led to an overall decrease in the content of both sennosides (Table 2) in the gelatin-encapsulated extracts, which is consistent with the capsule's low resistance to breakage (Table 1). Otherwise, a smaller decrease in their contents was observed when encapsulated with hypromellose shells, demonstrating the superior performance of this material over the gelatin concerning the stability of the chemical contents. A similar trend resulted from a highperformance thin-layer chromatography-based study on sennoside stability and different packaging materials ${ }^{[19]}$. Sennoside A and sennoside B have been shown to degrade according to distinct chemical pathways that involve (i) sugar hydrolysis (increasing the aglycone levels) or (ii) high-temperature induced oxidative decomposition ${ }^{[20]}$. Pathway (i) initially produces sennidin monoglycosides, which were detected by LC-MS in very low concentrations at the end of the experiments (data not shown). It is noteworthy that the sennoside B content decayed by approximately $10 \%$ in the gelatin capsules compared to the hypromellose (77\%) protection (Table 2). The higher lability of this species has also been reported from experiments involving increasing temperature and humidity on stored senna leaves and the methanolic extract obtained from them. Conditions varying from $25-40^{\circ}$ and 60 $75 \% \mathrm{RH}$ resulted in a sennoside content reduction that ranged from $12 \%$ to $90 \%$ for stored leaves after a one-year monitoring period. In the same period, extreme conditions $\left(40^{\circ}-75 \% \mathrm{RH}\right)$ led to complete sennoside decomposition in poorly packed extracts but only partial degradation when stored in wellconditioned bottles ${ }^{[20]}$. Although gelatin capsules have conventionally been used for commercial formulations of herbal materials, the present study demonstrates the superior performance of hypromellose capsules for maintaining the hardness and disintegration time of the capsules and humidity and sennoside content of formulations of senna dry extract after 6 mo at $30 \% 65 \% \mathrm{RH}$ and $40 \% / 75 \% \mathrm{RH}$. The significantly longest disintegration time for hypromellose capsules was already expected, as previously reported ${ }^{[17,21]}$. In addition, a possible effect of a fill material within the capsules on increasing of the disintegration time of capsule shells can be expected ${ }^{[17,21]}$. Conversely, the effect on disintegration time of capsules filled with plant dry extracts has not been observed so far. Water activity and susceptibility to microbial growth were compromised in the encapsulated extract on gelatin capsules, and the hypromellose capsules performed better than the gelatin capsules concerning other properties evaluated. Hypromellose capsules provided greater chemical stability of the senna dry extract than gelatin capsules, particularly with respect to the sennoside A and sennoside B content, which are quality chemical indicators for stability and pharmacological activity of senna extract. Overall, the results of this preliminary study indicate that hypromellose was advantageous over gelatin for building more stable senna extract-containing capsules, suggesting that it is a good alternative in the production of medicinal capsules containing herbal dry extracts, especially those with high hygroscopicity. Moreover, our results support the establishment of time and storage conditions for further stability studies of capsules containing these extracts. 


\section{Acknowledgements:}

The authors thank Capsugel (a division of Pfizer Laboratory) and the Program for Technological Development in Health Products (PDTIS/Fiocruz) for technical support.

\section{REFERENCES}

1. European Pharmacopoeia 9.0. Senna leaf dry extract, standardised - Monograph 07/2015:1261. Strasbourg: European Directorate for the Quality of Medicines \& HealthCare - Council of Europe; 2017.

2. Pachú CO. Processing of medicinal plants to obtain dry and liquid extracts [Processamento de plantas medicinais para obtenção de extratos secos e líquidos]. Doctorate thesis, Federal University of Campina Grande, Campina Grande; 2007.

3. Marques MRC, Cole E, Kruep D, Gray V, Murachanian D, Brown WE et al. Liquid-filled gelatin capsules. Pharmacopeial Forum 2009;35:1029-41.

4. Lachman L, Lieberman HA, Kaning JL. The theory and practice of industrial pharmacy. Philadelphia (PA): Lea \& Febiger; 1986.

5. Coni-Snap - hard gelatin capsules. Morristown (NJ): Capsugel; 2011. Available from: https://s3.amazonaws.com/cpsl-web/kc/ library/ConiSnap_brochure_full.pdf.

6. Vyas T, Dash RP, Anandjiwala S, Nivsarkar M. Formulation and pharmacokinetic evaluation of hard gelatin capsule encapsulating lyophilized Vasa Swaras for improved stability and oral bioavailability of vasicine. Fitoterapia 2011;82:44653.

7. Technical Reference File - Vcaps ${ }^{\circledR}$ Plus Capsules. 4th ed. Morristown: Capsugel; 2014. Available from: https:// s3.amazonaws.com/cpsl-web/kc/pdfs/library/VCaps_Plus_ Technical_Reference_Guide_Updated_March_2014.pdf.

8. Chong RHE, Jones BE, Díez F, Birchall JC, Coulman SA. Evaluating the sensitivity, reproducibility and flexibility of a method to test hard shell capsules intended for use in dry powder inhalers. Int J Pharm 2016;500:316-25.

9. Birchall JC, Jones BE, Morrissey A, Jones BE. A comparison of the puncturing properties of gelatin and hypromellose capsules for use in dry powder inhalers. Drug Dev Ind Pharm 2008;34:870-6.

10. Ku MS, Li W, Dulin W, Donahue F, Cade D, Benameur H et al. Performance qualification of a new hypromellose capsule: part I. Comparative evaluation of physical, mechanical and processability quality attributes of Vcaps Plus, Quali-V and gelatin capsules. Int J Pharm 2010;386:30-41.

11. Brazilian Pharmacopoeia. 5th ed. Vol. 1 - Chap. 5, General methods. Brasília: Anvisa \& Fiocruz; 2010.

12. United States Pharmacopoeia. USP 43-NF38. 〈731〉 Loss on drying and $\langle 1111\rangle$ Microbiological examination of nonsterile products: acceptance criteria. General Chapter. Rockville: US Pharmacopeial Convention; 2019. Consulted online in https:// online.uspnf.com/

13. ICH Expert Working Group. International Conference on Harmonisation of Technical Requirements for Registration of Pharmaceuticals for Human Use, ICH harmonised tripartite guideline, Guidance for Industry Q1A(R2) - Stability testing of new drug substances and products. Step 5 version. Geneva: ICH; 2003.

14. Association of Official Analytical Chemists. Official methods of analysis. 16th ed. Chap. 42, Water activity of canned vegetables. Gaithersburg (MD): AOAC International; 1995.

15. Bala S, Uniyal GC, Bubey T, Singh SP. An improved method for the analysis of sennosides in Cassia angustifolia by high-performance liquid chromatography. Phytochem Anal 2001; 12:277-80.

16. Digenis GA, Gold TB, Shah VP. Cross-linking of gelatin capsules and its relevance to their in vitro-in vivo performance. J Pharm Sci 1994;83:915-21.

17. El-Malah Y, Nazzal S, Bottom CB. Hard gelatin and hypromellose (HPMC) capsules: estimation of rupture time by real-time dissolution spectroscopy. Drug Dev Ind Pharm 2007;33:27-34.

18. ICH Expert Working Group. International Conference on Harmonisation of Technical Requirements for Registration of Pharmaceuticals for Human Use, ICH harmonised tripartite guideline, Guidance for Industry Q2(R1) - Validation of analytical procedures: text and methodology, Step 5 version. Geneva: ICH; 2005.

19. Upadhyay A, Chandel Y, Nayak PS, Khan NA. Sennoside contents in senna (Cassia angustifolia Vahl.) as influenced by date of leaf picking, packaging material and storage period. Journal of Stored Products and Postharvest Research 2011;2:97-103.

20. Goppel M, Franz G. Stability control of senna leaves and senna extracts. Planta Med 2004;70:432-6.

21. Missaghi S, Fassihi R. Evaluation and comparison of physicomechanical characteristics of gelatin and hypromellose capsules. Drug Dev Ind Pharm 2006;32:829-38. 